\title{
miR-141 confers docetaxel chemoresistance of breast cancer cells via regulation of EIF4E expression
}

\author{
YA-SAI YAO $^{1 *}$, WEN-SHENG QIU ${ }^{1 *}$, RU-YONG YAO ${ }^{2}$, QIAN ZHANG $^{2}$, \\ LI-KUN ZHUANG ${ }^{2}$, FEI ZHOU ${ }^{1}$, LI-BIN SUN ${ }^{1}$ and LU YUE ${ }^{1,3}$ \\ ${ }^{1}$ Department of Oncology, ${ }^{2}$ Central Laboratory and ${ }^{3}$ Tumor Molecular and Translational Medicine Laboratory, \\ Affiliated Hospital of Medical College, Qingdao University, Qingdao 266003, P.R. China
}

Received October 28, 2014; Accepted January 30, 2015

DOI: $10.3892 /$ or.2015.3866

\begin{abstract}
Resistance to docetaxel, a chemotherapy drug for breast cancer (BC) treatment, occurs in $\sim 50 \%$ of patients, and the underlying molecular mechanisms of drug resistance are not fully understood. Gene regulation through miR-141 has been proven to play an important role in cancer drug resistance. The present study investigated the role of miR-141 expression in BC cells of acquired docetaxel resistance. Inhibition of miR-141 enhanced the response to docetaxel in docetaxel-resistant cells (MCF-7/DTX and MDA-MB-231/DTX, respectively), whereas overexpression of miR-141 confered resistance in docetaxelsensitive cells (MCF-7 and MDA-MB-231, respectively). By directly targeting the eukaryotic translation initiation factor $4 \mathrm{E}$ (EIF4E) mRNA, miR-141 acts on genes that are necessary for drug induced apoptosis rendering the cells drug resistant. Modulation of miR-141 expression was correlated with EIF4E expression changes and a direct interaction of miR-141 with EIF4E was shown by a luciferase assay. Thus, the present study is the first to show an increased expression of miR-141 in an acquired model of docetaxel resistance in $\mathrm{BC}$. This serves as a mechanism of acquired docetaxel resistance in BC cells, possibly through direct interactions with EIF4E, therefore presenting a potential therapeutic target for the treatment of docetaxel resistant $\mathrm{BC}$.
\end{abstract}

\section{Introduction}

Breast cancer (BC) is the most frequently diagnosed cancer and the leading cause of cancer-related deaths in females worldwide, accounting for $23 \%$ (1.38 million) of the total new cancer cases and $14 \%(458,400)$ of the total cancer deaths in

Correspondence to: Professor Lu Yue, Department of Oncology, Affiliated Hospital of Medical College, Qingdao University, 16 Jiangsu Road, Qingdao 266003, P.R. China

E-mail: lu_yue11@yeah.net

${ }^{*}$ Contributed equally

Key words: miR-141, docetaxel, drug resistance, breast cancer, EIF4E
2008 (1). Even though treatment options have been greatly improved, resistance to classical chemotherapeutics is still a tremendous challenge for BC therapy (2). To date, there is no validated drug-response/resistant biomarker available in the clinical settings, and the underlying mechanisms of acquisition of resistance to chemotherapeutic agents are still poorly understood. Hence, an advancement of the treatment by avoiding drug resistance and a better prediction of chemotherapy efficacy would improve the clinical outcome for BC patients.

Several different mechanisms of chemoresistance in cancer have been elucidated. Changes in the intracellular metabolic machinery, induction of growth signaling and impairment of apoptosis all lead to the acquisition of drug resistance $(3,4)$. As a semisynthetic analogue of paclitaxel, docetaxel is widely used in the treatment of advanced BC and other solid tumors. It leads to cell cycle arrest and apoptosis by binding to and inhibiting the depolymerisation of the $\beta$-tubulin subunit of microtubules $(5,6)$. As with other chemotherapeutic agents, numerous patients are either intrinsically resistant or acquire resistance to docetaxel during the course of treatment.

The resistance to docetaxel results from both genetic and epigenetic dysregulation of key genes, involving drug transporters, changes in drug metabolism and pathway alterations of cell cycle and apoptosis (7-9). Identified as important post-transcriptional regulators, microRNAs (miRNAs) have expanded the definition of epigenetic regulation. miRNAs are endogenous, non-coding RNAs of $\sim 22$ nucleotides that target various genes either by degrading the mRNA or by repressing the translation (10). An altered expression of miRNAs in primary human types of cancers has been used for tumor diagnosis, classification, staging and prognosis (11). Moreover, they control the cell growth, proliferation, metabolism and apoptosis. In addition, the current research highlights their role in drug resistance in various types of cancers $(12,13)$.

As an oncogene or a tumor-suppressor gene, miR-141 has been reported to be either upregulated (ovarian and colorectal cancers) $(14,15)$ or downregulated (prostate, renal cell and hepatocellular carcinoma) (16-18) in various types of cancers. Recently, a study showed that downregulated miR-141 may confer cisplatin resistance in esophageal squamous cell carcinoma (19). Moreover, miR-141 modulates cisplatin sensivitity in ovarian cancer cells (20). Even though miR-141 has been intensively studied, no study has investigated the role of 
miR-141 expression in docetaxel-resistance in BC or the underlying mechanisms.

The present study is the first to report that increased miR-141 expression was associated with acquired docetaxel resistance in vitro and changes in miR-141 expression modulated response to docetaxel in $\mathrm{BC}$ cells, at least in part by targeting the eukaryotic translation initiation factor $4 \mathrm{E}$ (EIF4E). Furthermore, we showed that suppression of miR-141 or EIF4E significantly promoted or reduced docetaxel induced apoptosis, respectively. Our study revealed an increased expression of miR-141 in an acquired model of docetaxel resistance in breast cancer.

\section{Materials and methods}

Cell lines and culture. Human BC MCF-7 and MDA-MB-231 cell lines were obtained from ATCC. The parental cell line (termed docetaxel-sensitive cells) was step wide cultured and made resistant to docetaxel (termed docetaxel-resistant cells) following sequential exposure to docetaxel (SanofiAventis, Surrey, UK) at increasing concentrations as previously described (21). Cells were cultured and maintained in RPMI-1640 medium including L-glutamine, supplemented with $10 \%(\mathrm{v} / \mathrm{v})$ fetal calf serum and $1 \%(\mathrm{v} / \mathrm{v})$ penicillin/streptomycin (100,000 U/1 penicillin, $10 \mathrm{mg} / 1 \mathrm{streptomycin})$ at $37^{\circ} \mathrm{C}$ in a humidified atmosphere containing $5 \%$ carbon dioxide.

RNA isolation. Total RNA and miRNA fractions were isolated from the cell lines by TRIzol agent (Invitrogen, Carlsbad, CA, USA). The quality and quantity of the RNA was assessed with a NanoDrop ND-1000 Spectrophotometer (NanoDrop Technologies, Wilmington, DE, USA) at 260 and $280 \mathrm{~nm}$ (A260/280)

Real-time quantitative reverse transcription-PCR. For miRNA expression detection, reverse transcription (RT) reaction was performed with PrimeScript RT reagent kit and real-time quantitative RT-PCR (qRT-PCR) was performed using SYBR Premix Ex Taq ${ }^{\mathrm{TM}}$ II (both from Takara Biotechnology Co., Ltd., Dalian, China) on the basis of the protocol provided by the manufacturer. The expression of EIF4E mRNA was detected by quantitative PCR using paired primers. The designed PCR primers were as follows: EIF4E forward primer, 5'-ATGGCGACTGTCGAACCG-3' and reverse primer, 5'-ATTAGATTCCGTTTTCTCCTCTTC TG-3'; GAPDH forward primer, 5'-AAGGGAAGGTTGCT GGATAGG-3' and reverse primer, 5'-CACATCCACCTCCTC CACATC-3'. The expression of the target miRNA was normalized relative to that of the internal control, U6 and the expression of the target gene was normalized relative to the expression of glyceraldehyde-3-phosphate dehydrogenase (GAPDH), which was used as an internal control. Data were analyzed according to the comparative $\mathrm{Ct}$ method also referred to as the $2^{-\Delta \Delta C t}$ method.

miRNA mimics, miRNA inhibitors and siRNA. miR-141 mimics and inhibitors were purchased from Shanghai GenePharma Co. (Shanghai, China) along with the negative controls (miR-NC mimics or anti-miR-NC). siRNAs against EIF4E were designed and synthesized by RiboBio Co. (Guangzhou,
China). EIF4E siRNA oligonucleotide duplexes (or control siRNA) using Lipofectamine 2000 (Invitrogen) according to the manufacturer's protocol, respectively.

miR-141 transfection. miRNA-141 was overexpressed in MCF-7 or MDA-MB-231 docetaxel-sensitive cells using a miRNA-141 mimics. In MCF-7/DTX or MDA-MB-231/DTX docetaxel-resistant cells, miRNA-141 was knocked down using a miRNA-141 inhibitor. Cells $\left(1 \times 10^{6}\right)$ cultured in a well of a 6-well cell culture plate were transiently transfected with 50 pmol of miR-141 inhibitor and miR-141 mimics, and its negative control oligonucleotides. Transfected cells were resuspended and cultured in regular culture medium for 48-72 $\mathrm{h}$ before analysis.

Western blotting. Total cellular proteins were extracted by cell lysis, separated on an 8-10\% SDS-PAGE, transferred onto a PVDF membrane and incubated with primary antibodies against human EIF4E (Santa Cruz Biotechnology) at 1:500 overnight at $4^{\circ} \mathrm{C}$ and then with anti-rabbit IgG (horseradish peroxidase-conjugated secondary antibody) for $1 \mathrm{~h}$ at room temperature. After being washed, the membranes were developed with an ECL Plus Western Blotting Detection System (Amersham, UK). The loading control for western blotting was $\beta$-actin.

In vitro drug sensitivity assay. The sensitivity of the BC cells to docetaxel was evaluated using the 3-(4,5-dimethylthiazol2-yl)-2,5-diphenyltetrazolium bromide (MTT) assay as previously described (22). After 72-h culture, cell viability was assessed using the MTT assay. The absorbance of each well at $490 \mathrm{~nm}$ (A490) was read on a spectrophotometer. The concentration at which each drug produced $50 \%$ inhibition of growth $\left(\mathrm{IC}_{50}\right)$ was estimated by the relative survival curve. Four independent experiments were performed in quadruplicate.

Luciferase assay. The pGL3-EIF4E wild-type and pGL3EIF4E mutant constructs were constructed and either $400 \mathrm{ng}$ pGL3-EIF4E wild-type or pGL3-EIF4E mutant and $8 \mathrm{ng}$ pGL4.70 [hRluc] plasmid was co-transfected with $50 \mathrm{nM}$ miRNA-141 precursor. Transfected cells were incubated for $24 \mathrm{~h}$ before performing the dual luciferase assay according to the manufacturer's instructions (Promega). Firefly luciferase activity of the pGL3-control plasmid was normalized to the Renilla luciferase activity of pGL4.70 [hRluc]. Each assay was performed in triplicate.

Cell cycle and apoptosis analyses. At $24 \mathrm{~h}$ after miR-141 inhibitor transfection, cells were incubated with docetaxel for $48 \mathrm{~h}$. For cell cycle analysis, cells were trypsinized, pelleted and then resuspended in propidium iodide (PI) solution (Sigma-Aldrich, St. Louis, MO, USA), including $100 \mathrm{ng} / \mathrm{ml}$ of PI, $0.1 \mathrm{mg} / \mathrm{ml}$ of RNase A and $0.05 \%$ Triton X-100, for a 30 -min incubation at $37^{\circ} \mathrm{C}$ and analyzed by flow cytometry using a FACS calibur instrument with CellQuest Software (BD Biosciences, Franklin Lakes, NJ, USA).

The Annexin V-FITC Apoptosis Detection kit (BD Biosciences, La Jolla, CA, USA) was used for the apoptosis assays. Cells $\left(1 \times 10^{6}\right)$ were stained according to the manuf- 

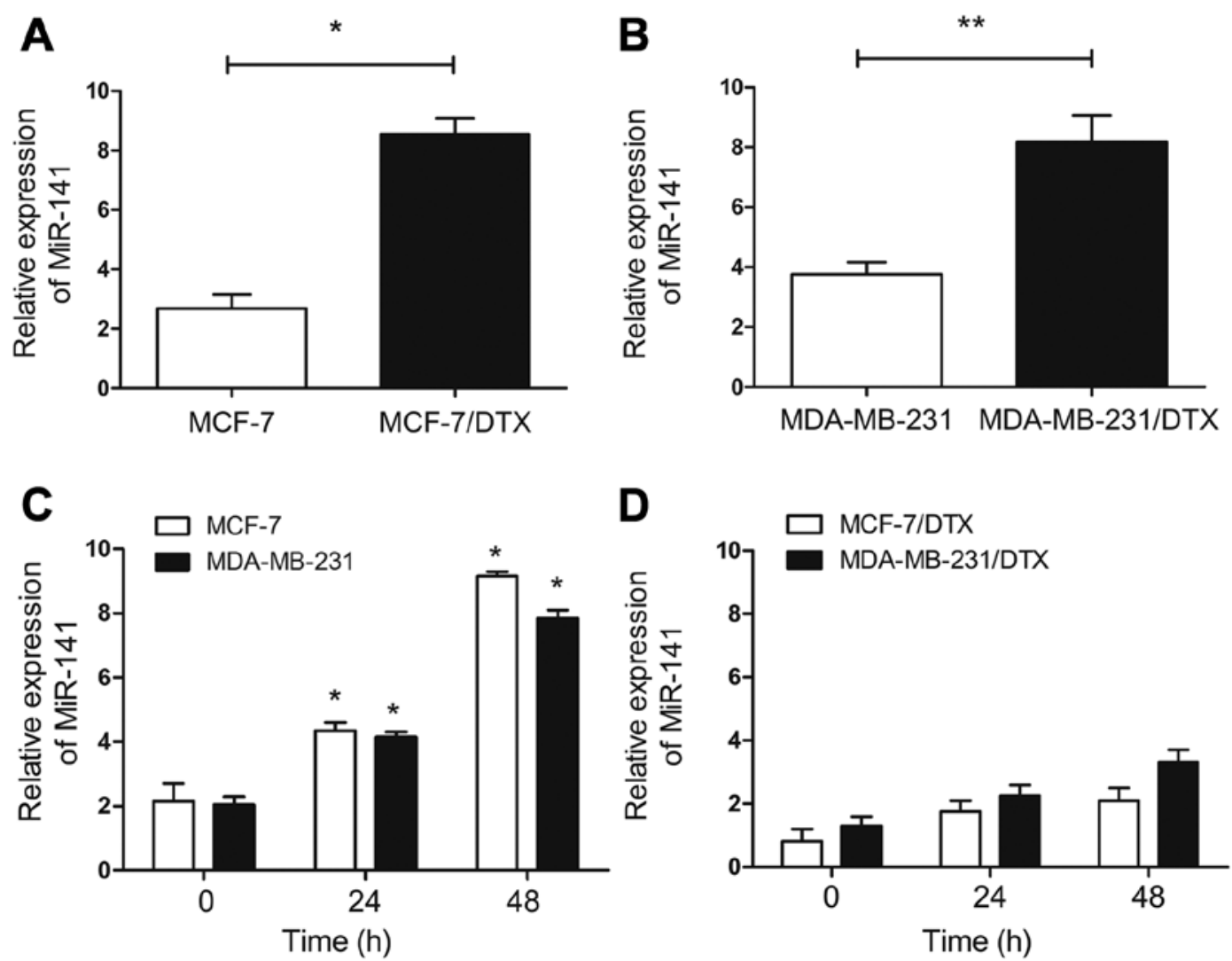

Figure 1. miR-141 expression in docetaxel-sensitive and -resistant human BC cell lines. (A and B) Relative expression levels of miR-141 were detected in parental (MCF-7 and MDA-MB-231) and resistant (MCF-7/DTX and MDA-MB-231/DTX) breast cancer cell lines via qRT-PCR. (C and D) Analysis of the expression of miR-141 in docetaxel-resistant or parental breast cancer cells after treatment with docetaxel (5 $\mu \mathrm{g} / 1)$ via qRT-PCR. ${ }^{*} \mathrm{P}<0.05,{ }^{* *} \mathrm{P}<0.01$. BC, breast cancer.

acturer's protocol and sorted using a FACS sorter and the data were analyzed using ModFit (both from BD Biosciences).

Bioinformatic and statistical analysis. Online miRNA databases (TargetScan, miRBase Targets and PicTarget) were used for prediction of miR-141 target genes. Each experiment was repeated at least 3 times. Numerical data are presented as the mean \pm SD. The difference between means was analyzed with ANOVA and then Student's t-test. All statistical analyses were performed using SPSS 19.0 software (SPSS, Inc., Chicago, IL, USA). Differences were considered to indicate a statistically significant result when ${ }^{*} \mathrm{P}<0.05$.

\section{Results}

miR-141 expression in docetaxel-sensitive and-resistant human BC cell lines. Two docetaxel-resistant BC cell lines (MCF-7/DTX and MDA-MB-231/DTX) were developed from the docetaxel-sensitive BC cell lines (MCF-7 and MDA-MB-231), respectively. To investigate whether miR-141 regulates the acquired docetaxel-resistance in $\mathrm{BC}$ cells, qRT-PCR was performed to detect the expression of miR-141 in docetaxel-resistant and parental BC cells. Notably, the results showed that miR-141 was expressed at significantly higher levels in the MCF-7/DTX and MDA-MB-231/DTX cells than that in their parental cells, respectively $(\mathrm{P}<0.05$, Fig. 1A; P<0.01, Fig. 1B).
Then, we further verified whether docetaxel treatment could affect the expression of miR-141 in BC cells. As shown in Fig. 1C and D, the miR-141 levels were increased in the parental MCF-7 or MDA-MB-231 cells after treatment with docetaxel $(5.0 \mu \mathrm{g} / \mathrm{l})$, but not in the MCF-7/DTX or MDA-MB-231/DTX cells.

Downregulation of miR-141 reverses the resistance of docetaxel-resistant BC cells to docetaxel. We further characterized the role of miR-141 in regulating chemotherapy resistance by modulating its levels in BC cells. Transient transfection of miR-141 inhibitor resulted in a significant decrease in docetaxel-resistant BC cells. As shown in Fig. 2A, the expression of miR-141 in miR-141 inhibitor-transfected MCF-7/DTX and MDA-MB-231/DTX cells was significantly decreased compared with that in the anti-miR-NC-transected cells $(\mathrm{P}<0.05)$. Further, the results of the MTT assay showed that miR-141 inhibitor decreased the growth of MCF-7/DTX and MDA-MB-231/DTX cells compared with anti-miR-NC cells (Fig. 2B).

Next, the changes of docetaxel sensitivity of chemoresistant BC cells induced by miR-141 inhibitor were analyzed. Compared with the anti-miR-NC-transfected cells, the $\mathrm{IC}_{50}$ value of docetaxel in the miR-141 inhibitor-transfected MCF-7/DTX and MDA-MB-231/DTX cells was significantly decreased by $\sim 44.0$ and $46.4 \%$, respectively ( $\mathrm{P}<0.05$, Fig. $2 \mathrm{C}$ ). From these experimental data, it was concluded that the 
A

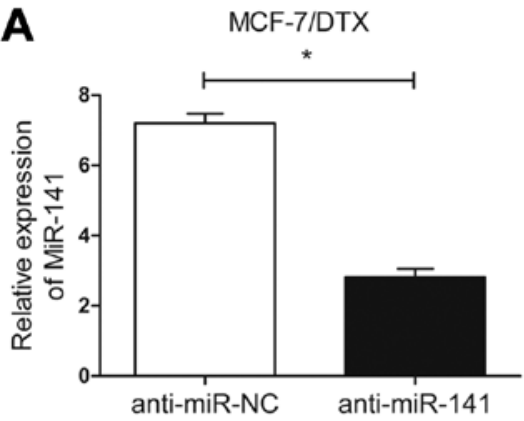

B

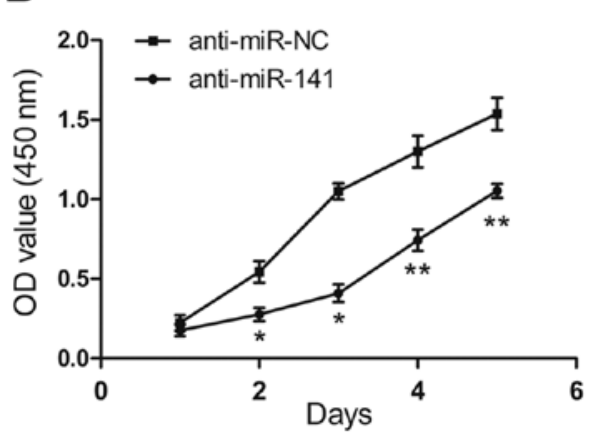

C

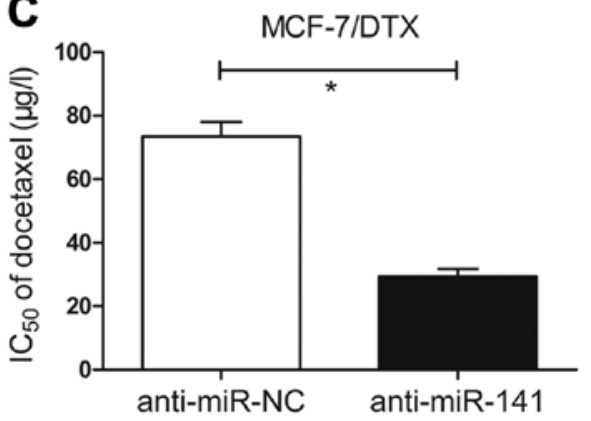

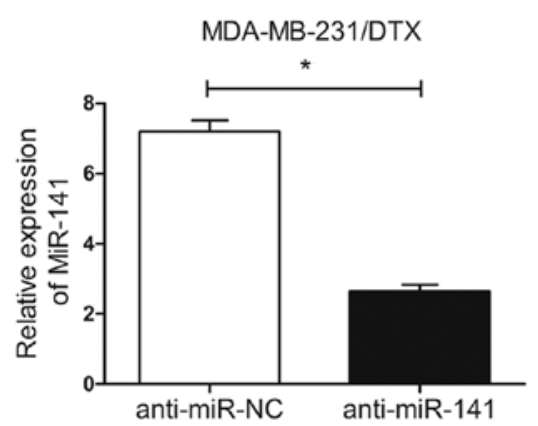
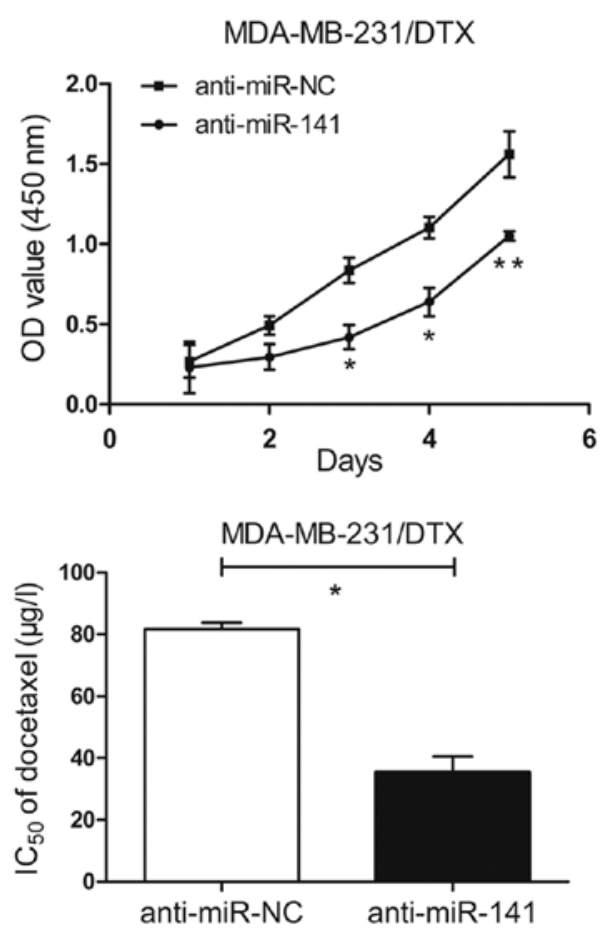

Figure 2. Downregulation of miR-141 reverses the resistance of docetaxel-resistant BC cells to docetaxel. (A) miR-141 expression levels were detected via qRT-PCR at $48 \mathrm{~h}$ post-transfection with miR-141 inhibitor and anti-miR-NC in MCF-7/DTX and MDA-MB-231/DTX cells. miRNA abundance was normalized to U6 RNA. (B) MTT analysis of growth in miR-141 inhibitor or anti-miR-NC-transfected MCF-7/DTX and MDA-MB-231/DTX cells. (C) Analysis of the $\mathrm{IC}_{50}$ values of docetaxel in MCF-7/DTX and MDA-MB-231/DTX cells that were transfected with anti-miR-NC or miR-141 inhibitor using MTT assays. ${ }^{*} \mathrm{P}<0.05,{ }^{* *} \mathrm{P}<0.01$ in comparison with anti-miR-NC-transfected cells.

downregulation of miR-141 could significantly reverse the resistance of docetaxel-resistant $\mathrm{BC}$ cells to docetaxel by inhibiting their growth and viability.

Upregulation of miR-141 reduces the in vitro sensitivity of parental BC cells to docetaxel. On the other hand, we investigated whether upregulation of miR-141 affected the sensitivity of BC cells to docetaxel. Then, miR-141 mimics were transfected into the parental MCF-7 or MDA-MB-231 cells. After $48 \mathrm{~h}$ transfection, qRT-PCR assay showed that the expression of miR-141 in the miR-141 mimic-transfected MCF-7 or MDA-MB-231 cells could be significantly increased $(\mathrm{P}<0.05$, Fig. 3A). MTT assay indicated that miR-141 mimics could induce the increased growth ability of the parental MCF-7 or MDA-MB-231 cells compared with miR-NC mimics (Fig. 3B). More importantly, the $\mathrm{IC}_{50}$ value of docetaxel in the miR-141 mimic-transfected MCF-7 or MDA-MB-231 cells were increased by $\sim 33.4$ or $42.3 \%$, respectively, compared with the miR-NC mimic-transfected cells $(\mathrm{P}<0.05$, Fig. $3 \mathrm{C})$. These data suggested that upregulation of miR-141 promotes the resistance of docetaxel in $\mathrm{BC}$ cells.

EIF4E is a target of miR-141 and responsible for the miR-141-induced resistance in BC cells. To examine the molecular mechanisms by which miR-141 regulates chemoresistance, we investigated its target genes and focused on a few that relate to cancer progression. By employing open access software (TargetScan, miRBase Targets and PicTarget), transcription factor EIF4E was chosen as a preferred candidate target gene of miR-141 due to the complementary site of miR-141 in its 3'-untranslated region (3'-UTR) (Fig. 4A). To investigate whether the EIF4E gene could exert functional effects in BC cells, western blot analysis was performed and downregulation of EIF4E protein expression was detected in the MCF-7/DTX and compared with MCF-7 cells. A similar trend was also found in the MDA-MB-231/DTX cells (Fig. 4B).

We investigated the correlation between miR-141 and EIF4E. As shown in Fig. 4C, compared with the NC-transfected 


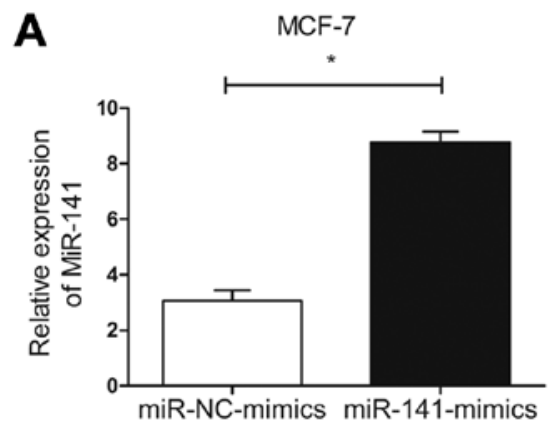

B

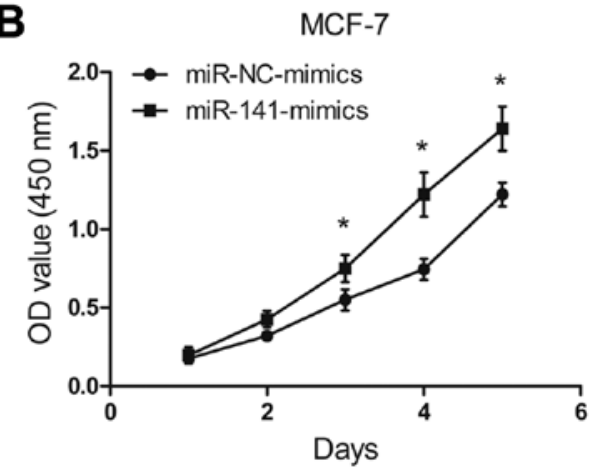

C

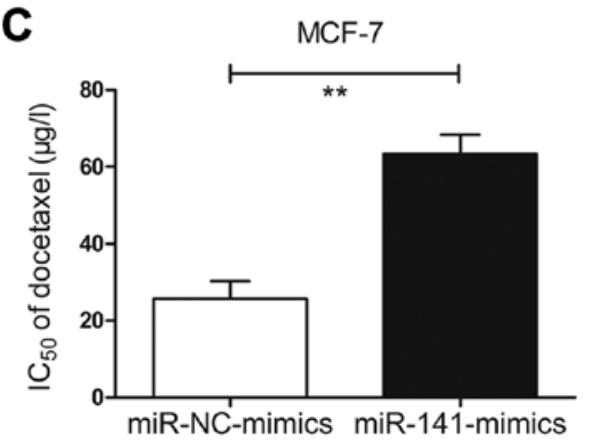

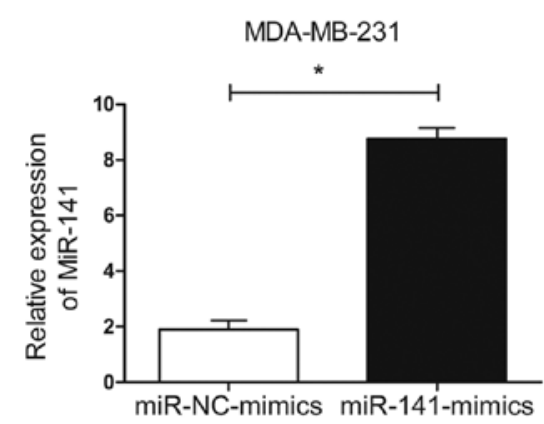

MDA-MB-231
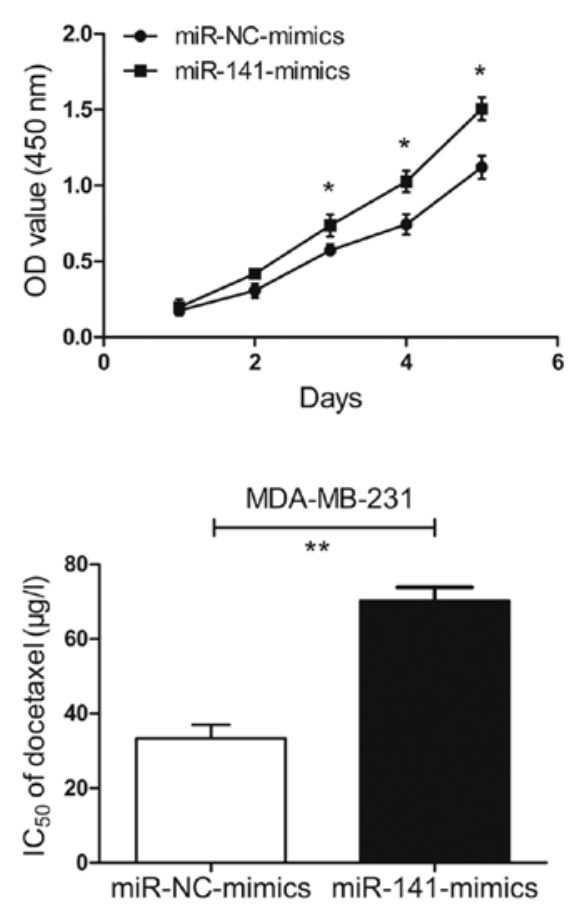

Figure 3. Upregulation of miR-141 reduces the in vitro sensitivity of parental BC cells to docetaxel. (A) miR-141 expression levels were detected via qRT-PCR at $48 \mathrm{~h}$ post-transfection with miR-141-mimics and miR-NC-mimics in MCF-7 and MDA-MB-231 cells. (B) MTT analysis of growth in miR-141-mimic or miR-NC-mimic-transfected MCF-7 and MDA-MB-231 cells. (C) Analysis of the $\mathrm{IC}_{50}$ values of docetaxel in MCF-7 and MDA-MB-231 cells transfected with miR-141-mimics and miR-NC-mimics using MTT assays. ${ }^{*} \mathrm{P}<0.05,{ }^{* * *} \mathrm{P}<0.01$ in comparison with miR-NC-mimic-transfected cells. BC, breast cancer.

cells, the level of EIF4E mRNA expression was significantly increased in the miR-141 inhibitor-transfected MCF-7/DTX and MDA-MB-231/DTX cells, and markedly decreased in the miR-141 mimic-transfected MCF-7 and MDA-MB-231 cells $(\mathrm{P}<0.05)$. While downregulation of the expression of EIF4E by siRNAs, MCF-7/MDA-MB-231 cells became more resistant to the therapy of docetaxel (Fig. 4D). In addition, the enhanced growth-inhibitory effect by the miR-141 inhibitor transfection was weakened after the addition of EIF4E siRNA (Fig. 4D). Those results suggest that the EIF4E expression is regulated by miR-141 in BC and is responsible for the miR-141-induced resistance.

To further confirm the possibility that miR-141 targets EIF4E, a dual luciferase reporter system was used to measure the direct interaction between miR-141 and EIF4E. Upon transfection of the miR-141 precursor, luciferase activity significantly decreased by 27.0 and $36.1 \%(\mathrm{P}<0.05)$ in the MCF-7/DTX/MDA-MB-231/DTX cells that contained the 3'-UTR with the miR-141 seed region of EIF4E, respectively (Fig. 4E). In contrast, addition of the miR-141 precursor did not alter luciferase activity in docetaxel-resistant cells that contained a mutated seed region in the 3'-UTR of the EIF4E sequence (Fig. 4E). By reducing luciferase activity, this shows that miR-141 directly interacts with the 3'-UTR region of the EIF4E gene.

Transfection of miR-141 inhibitor or siRNA-EIF4E promotes or reduces docetaxel-induced apoptosis, respectively. To explore the mechanism(s) by which miR-141 inhibitor induces sensitivity of BC cells to docetaxel, we analyzed the cell cycle and apoptosis alterations in MCF-7 cells transfected with the miR-141 inhibitor. Suppression of miR-141 had no significant alterations in the cell cycle (data not shown). We next measured cell apoptosis using an Annexin V/PI assay. The results showed a significant increase in the percentages of apoptosis of miR-141 inhibitor-transfected MCF-7 cells treated with docetaxel than the control cells (Fig. 5A, left panel) and significant decrease in the percentages of apoptosis of siRNA-EIF4E-transfected cells with docetaxel than the control cells (Fig. 5A, right panel). Therefore, the results 
A

Position 903-931

$$
\begin{aligned}
& \text { of EIF4E 3' UTR } \quad 5 \text { ' } \cdots \text { ccATTCATATTAAGACAGTGTac } \cdots 3 \text { ' } \\
& \text { ||:: ||:: |||||||| } \\
& \text { Hsa-miR-141 3' - ggUAGAAAUGG-UCUGUCACAau-5' }
\end{aligned}
$$

C
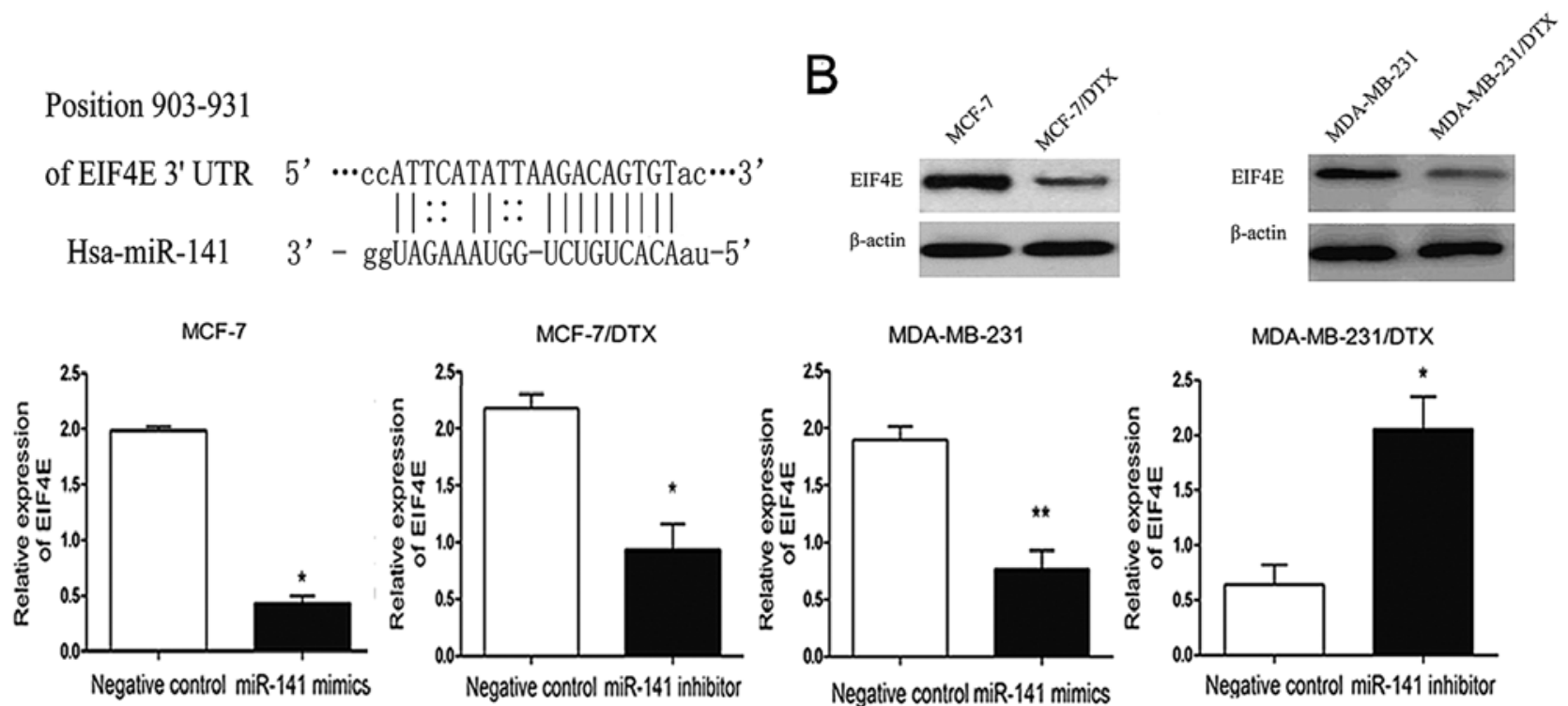

D

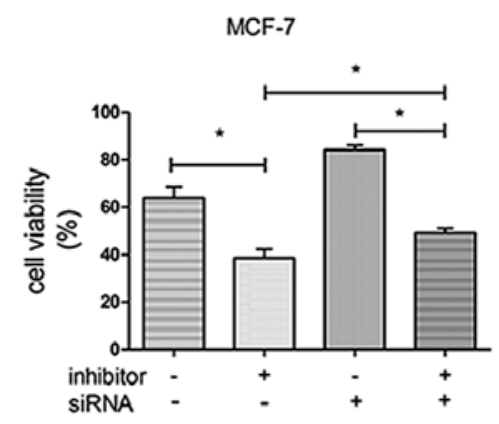

$\mathrm{E}$

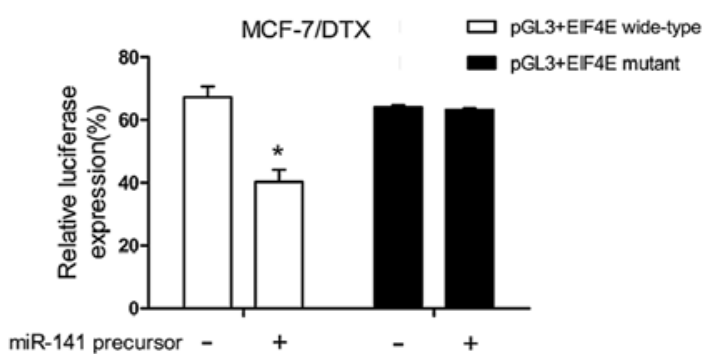

Negative control miR-141 mimics
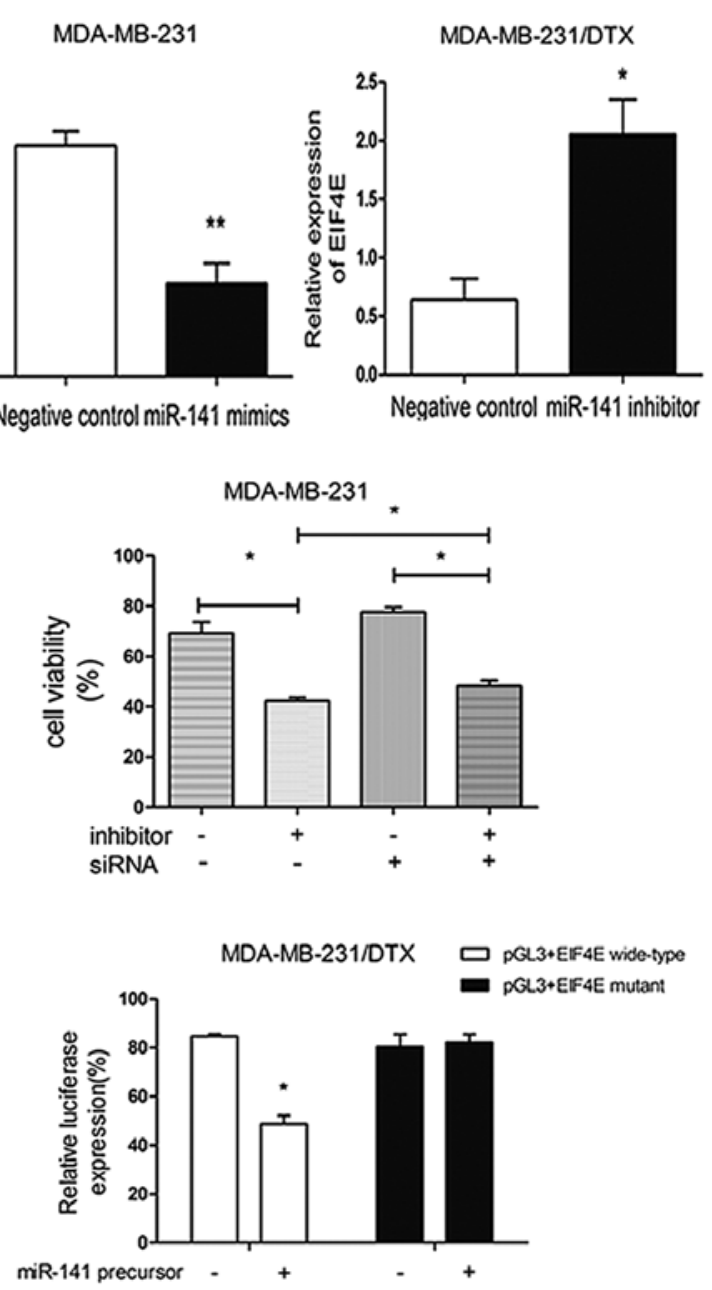

Figure 4. EIF4E is a target of miR-141 and responsible for the miR-141-induced resistance in BC cells. (A) The complementary sites of miR-141 in EIF4E 3'-UTR is shown as predicted by bioinformatics analysis. (B) Western blot analysis indicates that MCF-7/DTX and MDA-MB-231/DTX cells had a lower EIF4E protein expression level than that in parental cells. (C) qRT-PCR assay (at $48 \mathrm{~h}$ post-transfection) suggests a negative correlation between miR-141 and EIF4E expression at mRNA levels. EIF4E mRNA abundance was normalized to GAPDH. (D) Changes in the antitumor effects of docetaxel after transfection with the miR-141 inhibitor and/or siRNA against EIF4E in MCF-7 and MDA-MB-231 cells. (E) Relative luciferase levels were measured in docetaxel-resistant cells transfected with either pGL3+EIF4E wild-type plasmid or pGL3+EIF4E mutant plasmid and miR-141 precursor. Cells transfected with pGL3+EIF4E wild-type plasmid or pGL3+EIF4E mutant plasmid only served as control . ${ }^{*} \mathrm{P}<0.05,{ }^{* *} \mathrm{P}<0.01$. EIF4E, eukaryotic translation initiation factor 4E; BC, breast cancer; 3'-UTR, 3'-untranslated region.

suggested that downregulation of miR-141 could lead to an apoptosis-inducing effect similarly to that in upregulation of EIF4E.

Next, we measured the expression of apoptosis-related proteins in $\mathrm{BC}$ cells. As a result, treatment with docetaxel elevated the expression of Bax and lowered the expression of Bcl-2 in the miR-141 inhibitor-transfected cells compared to the mock-transfected cells (Fig. 5B). After treatment with docetaxel, decreased expression of Bax and increased Bcl-2 expression was noted when the cells were transfected with siRNA-EIF4E (Fig. 5C).

\section{Discussion}

The key finding of the present study is that miR-141 is correlated with breast cancer (BC) chemoresistance to docetaxel and serves as a phenotypic regulator of chemoresistance. While our studies focused on the role of miR-141 as a regulator of chemotherapy response, data showed that downregulation of miR-141 reversed the resistance of docetaxel-resistant BC cells. Furthermore, EIF4E was a target of miR-141 and partly responsible for the miR-141-induced resistance in BC cells. Transfection of miR-141 inhibitor and siRNA-EIF4E signifi- 

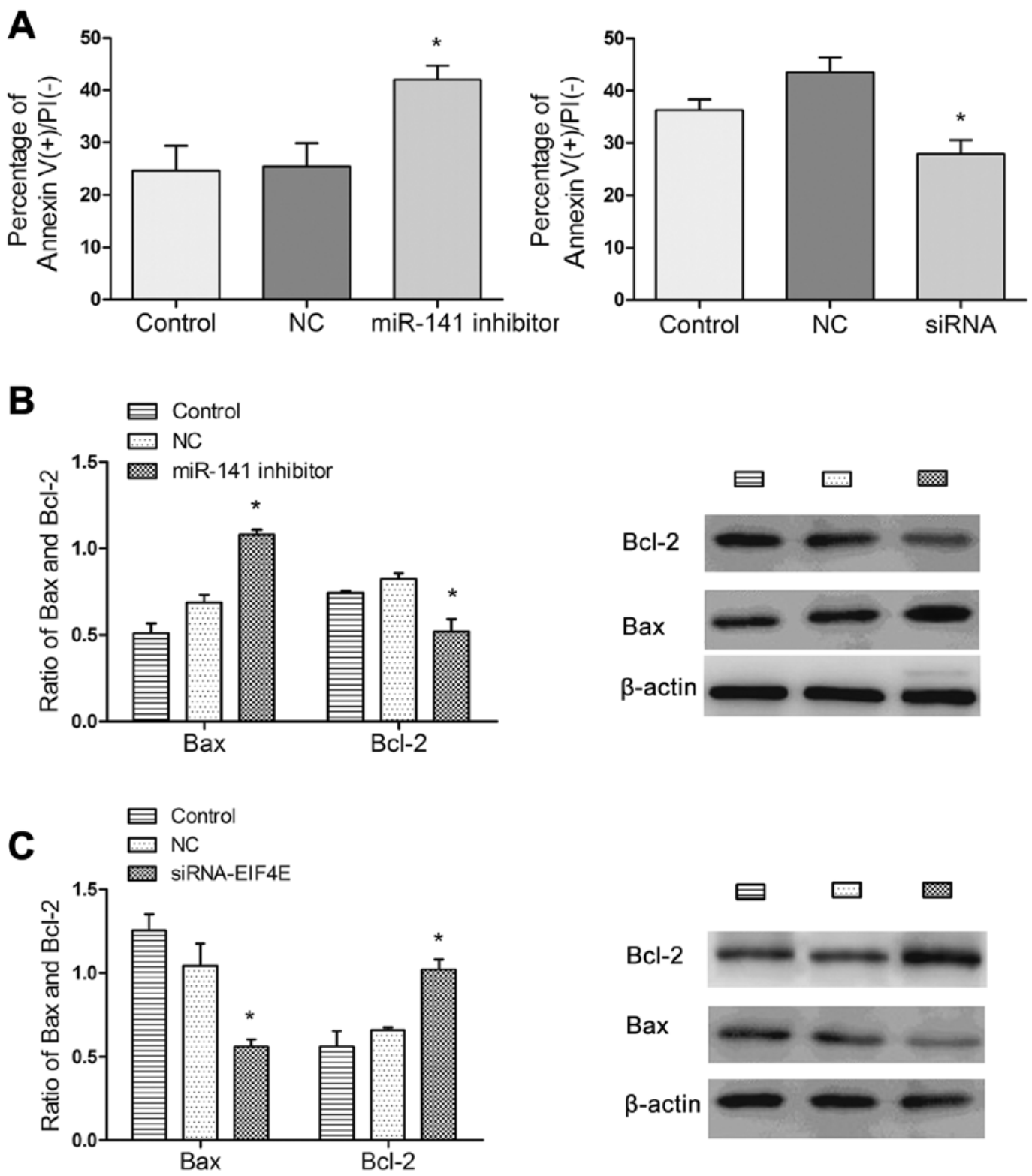

Figure 5. Transfection of the miR-141 inhibitor or siRNA-EIF4E promotes or reduces docetaxel-induced apoptosis. (A) Apoptosis analysis of BC cells after transfection of miR-141 inhibitor or siRNA against EIF4E. (B and C) Expression of apoptosis-related protein Bax and Bcl-2 in MCF-7 cells after transfection of miR-141 inhibitor and siRNA against EIF4E, respectively. ${ }^{*} \mathrm{P}<0.05,{ }^{* *} \mathrm{P}<0.01$. EIF4E, eukaryotic translation initiation factor $4 \mathrm{E}$; $\mathrm{BC}$, breast cancer.

cantly promoted docetaxel-induced apoptosis and changed the expression of Bcl-2 and BAX. These data suggest that miR-141 is a potential target for chemosensitizing BCs.

miR-141 is a widely studied human miRNA that was associated with cancer progression and metastasis in a variety of cancers (23-27) and recently, evidence emerged that miR-141 is also associated with drug resistance in cancer. Zhang et al reported that miR-141 can predict tumor progression in prostate cancer (28). Also, high miR-141 plasma level is associated with poor prognosis in colorectal cancer and was proposed as a novel biomarker to find distant metastases (15). All the above research reported that miR-141 not only to acts as a tumor suppressor, but also as an oncogene. Equally important, Imanaka et al highlighted an important regulatory role for miR-141 in the development of cisplatin resistance in esophageal squamous cell carcinoma (19). Furthermore, the miR-141-mediated regulation of KEAP1 has a crucial role in the cellular response to cisplatin in ovarian cancer cells (20). Moreover, the downregulated miR-141 was involved in Helicobacter pylori-modulated cisplatin sensitivity in gastric cancer (29). However, there are no previous reports concerning the association of miR-141 expression with the chemoresistance of BC tumor cells. In the present study, we firstly showed that miR-141 was significantly upregulated in docetaxel-resistant BC cells (MCF-7/DTX and MDA-MB-231/DTX) compared with parental $\mathrm{BC}$ cells. By modulating the miR-141 level in $\mathrm{BC}$ cells, we revealed that miR-141 mediated the docetaxel sensitivity of BC cells in vitro, which was consistent with the majority of the above studies.

Although several studies identified the role of miR-141 in chemoresistance, the underlying mechanism of the miR-141induced chemoresistance was still unclear. Most miRNAs function through the inhibition of effective mRNA translation of target genes, which may be involved in the progression of 
cancer and chemoresistance. Previous research showed that the NF- $\mathrm{NB}$ pathway, which can be regulated by KEAP1, is activated upon miR-141 overexpression, and the inhibition of this pathway partially reverses miR-141-mediated cisplatin resistance in ovarian cancer (29). van Jaarsveld et al, stressed that miR-141 is an oncogene that can enhance anoikis resistance in ovarian cancer cells through downregulation of KLF12. Indeed, the known targets of miR-141, KEAP1 and KLF12 played an important role in regulating drug-resistance and cancer development (20).

Eukaryotic initiation factor 4E (EIF4E), is a eukaryotic translation initiation factor involved in directing ribosomes to the cap structure of mRNAs and plays an important role in the development and progression of cancer $(30,31)$. It contributes to malignancy by selectively enabling the translation of a limited pool of mRNAs that encode key proteins involved in cell proliferation, angiogenesis and survival, as well as in transformation and metastasis $(32,33)$. Increased eIF4E expression has been shown in several cancer cells (including those of the breast, head and neck, bladder, colon, lung and prostate) (34-36) and its overexpression also appears to be related to disease progression (37). Moreover, studies showed that cellular overexpression of eIF4E results in increased proliferation and suppression of apoptosis, whereas reduction in eIF4E expression, reduces tumorigenesis (38). In our previous study, we demonstrated that siRNA-mediated EIF4E knockdown yielded very similar effects as that of ectopic miR-141 expression in BC cells. EIF4E is responsible for the miR-141 modulating chemosensitivity of BC cells to docetaxel and it was a direct target of miR-141. Moreover, EIF4E is partly responsible for the miR-141-induced apoptosis. This suggests that miR-141-mediated docetaxel resistance occurs through regulation of EIF4E and EIF4E is involved in the regulation of the apoptosis process. Therefore, the biological role of decreased EIF4E in docetaxel resistance remains unclear and can only be speculated upon. The EIF4E family contains a variety of genes which may also be a target of miR-141. Therefore, alterations of miR-141 may trigger the apoptotic gene changes, whereby EIF4E expression is predominated by regulating the expression of other anti-apoptotic genes, which in turn may lead to resistance to docetaxel. It was concluded that downregulation of EIF4E may be a molecular mechanism by which miR-141 exerted its functions of chemosensitivity regulator on human $\mathrm{BC}$ cells.

Furthermore, we showed that transfection of miR-141 inhibitor could significantly promote docetaxel-induced apoptosis and change the expression of Bax and Bcl-2. However, when the BC cells were transfected with siRNAEIF4E, the data showed opposite results. It suggested that EIF4E is partly responsible for the miR-141-induced apoptosis which is related to the mitochondrial apoptosis pathway. In the previous studies, antisense Bcl-2 treatment enhanced sensitivity to tamoxifen in HER2-positive cells in tamoxifenresistant $\mathrm{BC}$ cells (39). In addition, $\mathrm{Bcl}-2$ overexpression, causes paclitaxel resistance in MCF-7 cells (40). These studies showed, that even though Bcl-2 is known as an anti-apoptotic protein, increased $\mathrm{Bcl}-2$ expression can be beneficial in $\mathrm{BC}$ treatment, whereas decreased $\mathrm{Bcl}-2$ expression is associated with drug resistance or poorer outcome. In the present study, the mechanisms by which the decreased $\mathrm{Bcl}-2$ expression negatively influenced the activity of docetaxel are currently not known. The apoptotic pathway is a complex machinery that involves a variety of apoptotic genes, therefore it may be possible that other factors influence the cell to enter or evade docetaxel-induced apoptosis.

In conclusion, the results of the present study demonstrated that miR-141 affected the chemosensitivity of BC cells to docetaxel by directly targeting EIF4E, due to its anti-apoptotic properties. Our data suggest that miR-141 may serve as a potential target for BC therapy. The study highlights the potentially important role of miR-141 in the development of drug resistance, and suggests that miR-141 may serve as biomarkers for response to chemotherapy. Further investigation of a larger patient population will be necessary to confirm the association of miR-141 and its target EIF4E with the responses of BC patients to docetaxel-based chemotherapy.

\section{Acknowledgements}

The present study was supported by the Shandong Natural Science Foundation (Y2006C23).

\section{References}

1. Jemal A, Bray F, Center MM, Ferlay J, Ward E and Forman D: Global cancer statistics. CA Cancer J Clin 61: 69-90, 2011.

2. Chuthapisith S, Eremin JM, El-Sheemy M and Eremin O: Neoadjuvant chemotherapy in women with large and locally advanced breast cancer: chemoresistance and prediction of response to drug therapy. Surgeon 4: 211-219, 2006.

3. Liu Y, Du F, Chen W, Yao M, Lv K and Fu P: Knockdown of dual specificity phosphatase 4 enhances the chemosensitivity of MCF-7 and MCF-7/ADR breast cancer cells to doxorubicin. Exp Cell Res 319: 3140-3149, 2013.

4. Li M, Yin J, Mao N and Pan L: Upregulation of phosphorylated cofilin 1 correlates with taxol resistance in human ovarian cancer in vitro and in vivo. Oncol Rep 29: 58-66, 2013

5. Yvon AM, Wadsworth P and Jordan MA: Taxol suppresses dynamics of individual microtubules in living human tumor cells. Mol Biol Cell 10: 947-959, 1999.

6. Wang TH, Wang HS and Soong YK: Paclitaxel-induced cell death: where the cell cycle and apoptosis come together. Cancer 88: 2619-2628, 2000.

7. Hopper-Borge E, Chen ZS, Shchaveleva I, Belinsky MG and Kruh GD: Analysis of the drug resistance profile of multidrug resistance protein 7 (ABCC10): resistance to docetaxel. Cancer Res 64: 4927-4930, 2004.

8. Rouzier R, Rajan R, Wagner P, et al: Microtubule-associated protein tau: a marker of paclitaxel sensitivity in breast cancer. Proc Natl Acad Sci USA 102: 8315-8320, 2005.

9. Kastl L, Brown I and Schofield AC: miRNA-34a is associated with docetaxel resistance in human breast cancer cells. Breast Cancer Res Treat 131: 445-454, 2012.

10. Bartel DP: MicroRNAs: genomics, biogenesis, mechanism, and function. Cell 116: 281-297, 2004.

11. Voorhoeve PM: MicroRNAs: oncogenes, tumor suppressors or master regulators of cancer heterogeneity? Biochim Biophys Acta 1805: 72-86, 2010.

12. Hao J, Zhao S, Zhang Y, et al: Emerging role of microRNAs in cancer and cancer stem cells. J Cell Biochem 115: 605-610, 2014.

13. Blower PE, Chung JH, Verducci JS, et al: MicroRNAs modulate the chemosensitivity of tumor cells. Mol Cancer Ther 7: 1-9, 2008.

14. Iorio MV, Visone R, Di Leva G, et al: MicroRNA signatures in human ovarian cancer. Cancer Res 67: 8699-8707, 2007.

15. Yin J, Bai Z, Song J, et al: Differential expression of serum miR-126, miR-141 and miR-21 as novel biomarkers for early detection of liver metastasis in colorectal cancer. Chin J Cancer Res 26: 95-103, 2014.

16. Porkka KP, Pfeiffer MJ, Waltering KK, Vessella RL, Tammela TL and Visakorpi T: MicroRNA expression profiling in prostate cancer. Cancer Res 67: 6130-6135, 2007. 
17. Chen $X$, Wang $X$, Ruan A, et al: miR-141 is a key regulator of renal cell carcinoma proliferation and metastasis by controlling EphA2 expression. Clin Cancer Res 20: 2617-2630, 2014.

18. Liu Y, Ding Y, Huang J, et al: MiR-141 suppresses the migration and invasion of HCC cells by targeting Tiam1. PLoS One 9: e88393, 2014.

19. Imanaka Y, Tsuchiya S, Sato F, Shimada Y, Shimizu K and Tsujimoto G: MicroRNA-141 confers resistance to cisplatininduced apoptosis by targeting YAP1 in human esophageal squamous cell carcinoma. J Hum Genet 56: 270-276, 2011.

20. van Jaarsveld MT, Helleman J, Boersma AW, et al: miR-141 regulates KEAP1 and modulates cisplatin sensitivity in ovarian cancer cells. Oncogene 32: 4284-4293, 2013.

21. Brown I, Shalli K, McDonald SL, et al: Reduced expression of p27 is a novel mechanism of docetaxel resistance in breast cancer cells. Breast Cancer Res 6: R601-R607, 2004

22. Juengel E, Nowaz S, Makarevi J, et al: HDAC-inhibition counteracts everolimus resistance in renal cell carcinoma in vitro by diminishing cdk2 and cyclin A. Mol Cancer 13: 152, 2014.

23. Zhao G, Wang B, Liu Y, et al: miRNA-141, downregulated in pancreatic cancer, inhibits cell proliferation and invasion by directly targeting MAP4K4. Mol Cancer Ther 12: 2569-2580, 2013.

24. Mateescu B, Batista L, Cardon M, et al: miR-141 and miR-200a act on ovarian tumorigenesis by controlling oxidative stress response. Nat Med 17: 1627-1635, 2011.

25. Morales-Prieto DM, Schleussner E and Markert UR: Reduction in miR-141 is induced by leukemia inhibitory factor and inhibits proliferation in choriocarcinoma cell line JEG-3. Am J Reprod Immunol 66 (Suppl 1): S57-S62, 2011.

26. Zhang L, Deng T, Li X, et al: microRNA-141 is involved in a nasopharyngeal carcinoma-related genes network. Carcinogenesis 31 559-566, 2010

27. Du Y, Xu Y, Ding L, et al: Down-regulation of miR-141 in gastric cancer and its involvement in cell growth. J Gastroenterol 44 556-561, 2009

28. Zhang HL, Qin XJ, Cao DL, et al: An elevated serum miR-141 level in patients with bone-metastatic prostate cancer is correlated with more bone lesions. Asian J Androl 15: 231-235, 2013.
29. Zhou X, Su J, Zhu L and Zhang G: Helicobacter pylori modulates cisplatin sensitivity in gastric cancer by down-regulating miR-141 expression. Helicobacter 19: 174-181, 2014.

30. Sonenberg N: eIF4E, the mRNA cap-binding protein: from basic discovery to translational research. Biochem Cell Biol 86 178-183, 2008.

31. Thoreen CC: Many roads from mTOR to eIF4F. Biochem Soc Trans 41: 913-916, 2013

32. Fischer PM: Cap in hand: targeting eIF4E. Cell Cycle 8 2535-2541, 2009.

33. Mathonnet G, Fabian MR, Svitkin YV, et al: MicroRNA inhibition of translation initiation in vitro by targeting the cap-binding complex eIF4F. Science 317: 1764-1767, 2007.

34. Graff JR and Zimmer SG: Translational control and metastatic progression: enhanced activity of the mRNA cap-binding protein eIF-4E selectively enhances translation of metastasis-related mRNAs. Clin Exp Metastasis 20: 265-273, 2003.

35. Nasr Z, Robert F, Porco JA Jr, Muller WJ and Pelletier J: eIF4F suppression in breast cancer affects maintenance and progression. Oncogene 32: 861-871, 2013.

36. Furic L, Rong L, Larsson O, et al: eIF4E phosphorylation promotes tumorigenesis and is associated with prostate cancer progression. Proc Natl Acad Sci USA 107: 14134-14139, 2010.

37. De Benedetti A and Graff JR: eIF-4E expression and its role in malignancies and metastases. Oncogene 23: 3189-3199, 2004.

38. Avdulov S, Li S, Michalek V, et al: Activation of translation complex eIF4F is essential for the genesis and maintenance of the malignant phenotype in human mammary epithelial cells. Cancer Cell 5: 553-563, 2004

39. Kim R, Tanabe K, Emi M, Uchida Y and Toge T: Modulation of tamoxifen sensitivity by antisense Bcl-2 and trastuzumab in breast carcinoma cells. Cancer 103: 2199-2207, 2005.

40. Huang Y, Ray S, Reed JC, et al: Estrogen increases intracellular p26Bcl-2 to p21Bax ratios and inhibits taxol-induced apoptosis of human breast cancer MCF-7 cells. Breast Cancer Res Treat 42: 73-81, 1997. 